\title{
MATERNAL HEIGHT AS AN INDEPENDENT RISK F ACTOR FOR NEONATAL SIZE AMONG ADOLESCENT BENGALEES IN KOLKATA, INDIA
}

\author{
Samiran Bisai (MSc, PhD, MIPHA(India)) ${ }^{1,2}$
}

\begin{abstract} of baby was lower in short mother than their counterparts.

\section{INTRODUCTION}

Low Birth Weight (LBW; birth weight $<2500 \mathrm{~g}$ ) (1) is a major public health problem in developing countries (2) particularly in the Indian subcontinent, where the LBW with $30-50 \%$ LBW rate, which are among the highest in the world (3). The causes of LBW are multifactorial (4-6), associated with medical complication in pregnancy, genetic, environmental, socio-cultural, demographic and nutritional variables. In addition, other factors related with maternal anthropometry (7) such as maternal weight, height, BMI, arm circu mference and head circumference have impact (8).
\end{abstract}

BACKGROUND: Low Birth Weight is a major public health problem in developing countries. The causes of LBW are multifactorial including complication during pregnancy, genetic, environmental, social-cultural, demographic and nutritional variables. Comparison of anthropometric risk factors for neonatal size of adolescent mothers are lacking from West Bengal. Therefore, this study was undertaken to identify maternal anthropometric characteristics, which most strongly influence neonate weight and length among Bengalee mothers.

METHODS: A hospital based cross-sectional study was undertaken during 2004 in a Government general hospital in South Kolkata, India. A total of 76 adolescent (age<20years) pregnant women were enrolled from obstetric ward who were admitted for delivery. Due to 4 perinatal deaths; a total of 72 adolescent mother-baby pairs were included in this analysis. Anthropometric measurements were undertaken immediately after delivery following stabilization as well maternal body mass index (BMI) was calculated using standard formula.

RESULTS: The prevalence of low birth weight $(\mathrm{LBW})$ in the present study was $52.8 \%$. The results revealed that $30.6 \%$ of mothers were undernourished $\left(B M I<19.8 \mathrm{~kg} / \mathrm{m}^{2}\right)$. It was noted that about $64 \%$ of undernourished mothers delivered $L B W$ baby. Linear regression analyses of neonatal weight and length as dependent variables revealed that in both cases, maternal height had the most significant impact. It showed $12.9 \%$ (birth weight) and $16.1 \%$ (birth length) of variation. Moreover, the proportion of $L B W$ was $75 \%, 52.3 \%$ and $25 \%$ among short (height $\leq 145 \mathrm{~cm}$ ), average $(146-155 \mathrm{~cm})$ and tall $(>155 \mathrm{~cm})$ mothers $\left(x^{2}=6.855, p<0.01\right)$, respectively. Short mothers had 2.74 and 9.0 fold greater risk of delivering $L B W$ baby than average and tall mothers. In contrast, mean birth weight and length

CONCLUSION: This study revealed that maternal height had the strongest significant impact on neonate size. This strong association could have serious health implications for Bengalee adolescent mothers. However, since this is a preliminary finding, it needs validation using a larger sample of adolescent mothers.

KEYWORDS: India; Bengalee; adolescents; maternal; neonates; Kolkata

It was well documented that malnourished mothers are more prone to have LBW babies (4) and pregnancy complications (9). Generally, malnourished girls tend to have short stature in adulthood and they have high rates of adverse pregnancy outcome such as perinatal mortality and prematurity (10). Several studies from India observed very high rates of LBW babies among mothers with height less than $140 \mathrm{~cm}$ (11-13). Another study reported $216 \mathrm{~g}$-birth weight variation between short $(<143 \mathrm{~cm})$ and tall $(>162 \mathrm{~cm})$ mothers $(6)$ and Bhatia et al (14) noticed birth weight increased as maternal height increased. Moreover, WHO collaborative study of maternal anthropometry and pregnancy outcome recommends the use of maternal height and weight for screening in its service application (7).

\footnotetext{
${ }^{1}$ Department of Anthropology, Vidyasagar University, Midnapore, West Bengal, India.

${ }^{2}$ Society for Applied Studies, CF -198, Sector - I, Salt Lake City, Kolkata - 700 064, West Bengal, India. Address for Correspondence: Dr. Samiran B isai, Department of Anthropology, Vidy asagar University, Midnapore Pin Code - 721 102, West Bengal, India. Phone: +91-9563140058, 9434021821, E-mail: samiranbisai@yahoo.com
} 
The causes and consequences of LBW in different ethnic groups are of much interest to epidemiologists, health care workers, human biologists and biological anthropologists. However, studies that have compared these risk factor profiles of teenage mothers are lacking from West Bengal. Moreover, to the best of the author's knowledge there exists no published report, which deals with the association of maternal anthropometric characteristics and neonatal size among adolescent Bengalee mothers.

In view of this, this study was undertaken to identify maternal anthropometric characteristics, which most strongly influence neonate weight and length among Bengalee mothers.

\section{SUBJECTS AND METHODS}

A hospital based cross-sectional study was undertaken during 2004 in a Government general hospital in south Kolkata, India. This hospital serves the needs of patients belonging to the lower - middle class socioeconomic group. The estimated minimum sample size $(n=72)$ was calculated using a formula to determine sample size for single proportion population, taking the prevalence (p) of LBW 23\% in the same hospital (15) and desired precision of $10 \%$ at the $95 \%$ confidence interval. All together 76 adolescent (age $<20$ years) pregnant women were enrolled randomly three days in a week from obstetric ward those who were admitted for delivery. The mothers and baby pairs who were not suffering from any significant medical and surgical disorders at the time of enrollment were included. For cases, birth data was not collected for four cases of perinatal death. Therefore, a total of 72 adolescent mother- baby pairs were included for the present analyses.

Monthly family income was recorded to the nearest year and Rupee (Rs.), respectively. All anthropometric measurements were undertaken immediately (within 24 hours) after delivery following stabilization using standard protocol (16). Mother weight and height was made and recorded to the nearest $100 \mathrm{~g}$ and $0.1 \mathrm{~cm}$, respectively, using Salter digital weighing scale and anthropometer rod. Similarly, head circumference and mid upper arm circumference was measured to the precision of $0.1 \mathrm{~cm}$ using non-stretchable fibre tape. Biceps and triceps skin fold thickness was measured using Lange skin-fold caliper to the nearest $0.2 \mathrm{~mm}$, respectively. Maternal body mass index (BMI) was calculated using standard formula. While, newborn birth weight and length was measured by using triple beam balance and locally made neonatometer to the nearest $1 \mathrm{~g}$ and $0.1 \mathrm{~cm}$, respectively.
Ethical Committee of the Society for Applied Studies, Kolkata, approved the study protocol. A written consent was obtained from each mother. Information on age, ethnicity, years of formal schooling, maternal obstetric, age, family size and monthly income were obtained using a questionnaire.

The distributions of continuous variables were not significantly skewed thus, not necessitating their transformation. Linear regression analyses were used to test for the impact of independent variables. Neonate birth length and weight were used as dependent variables. Odds ratio (OR) and 95\% confidence interval (CI) was calculated using standard formula. All statistical analyses were performed using SPSS for windows version 7.5. Statistical significance was considered at $\mathrm{p}<0.05$.

\section{RES ULTS}

The average monthly family income was Rupees 3665.30 ( $S D \pm 2166.7$ ). It must be noted here that the exchange rate during the study period was 46 rupees to approximately 1 USD. The mean age and gestational age were 18.3 (0.8) years and 38.1 (2.4) weeks, respectively. The mean family size and formal years of schooling were 4.7 (2.4) person and 5 (3.3) years, respectively.

Anthropometric characteristics of maternal and neonates expressed as means, standard deviation, minimum and maximum values are presented in Table 1. The mean (SD) of weight, height, head circumference, MUAC, biceps and triceps skin-fold were 46 (5.9) kg, 149.4 (6) cm, 51.9 (1.5) cm, 22.5 (1.9) $\mathrm{cm}, 3.7(1.3) \mathrm{mm}$ and $8.6(3.2) \mathrm{mm}$, respectively. The mean (SD) BMI of mothers' was $20.6(2.2) \mathrm{kg} / \mathrm{m}^{2}$. Moreover, Mean birth weight and birth length baby was 2474 (409) gram and $47.3(2.1) \mathrm{cm}$, respectively.

The prevalence of low birth weight (birth weight $<2.5 \mathrm{~kg}$ ) was $52.8 \%$. The results revealed that $30.6 \%$ of mothers were undernourished (BMI $<19.8$ $\mathrm{kg} / \mathrm{m}^{2}$ ) and about $64 \%$ of undernourished mothers delivered LBW baby. Moreover, the proportion of LBW was $75 \%, 52.3 \%$ and $25 \%$ among short (height $\leq 145 \mathrm{~cm})$, average $(146-155 \mathrm{~cm})$ and tall mothers $(>155 \mathrm{~cm})$. Short mothers had 2.74 (95\%CI: 0.6712.04) and 9 (95\% CI: 1.24-78.65) fold greater risk of being LBW baby than average and tall mothers. There was a significantly decreasing trend with advancement of maternal height $\left(\mathrm{x}^{2}=6.855, \mathrm{p}<0.01\right)$. In contrast, mean birth weight and length of baby was lower for short mothers, mean birth weight (figure 1) and length of baby (figure 2) was significantly increased with increment of maternal height. 


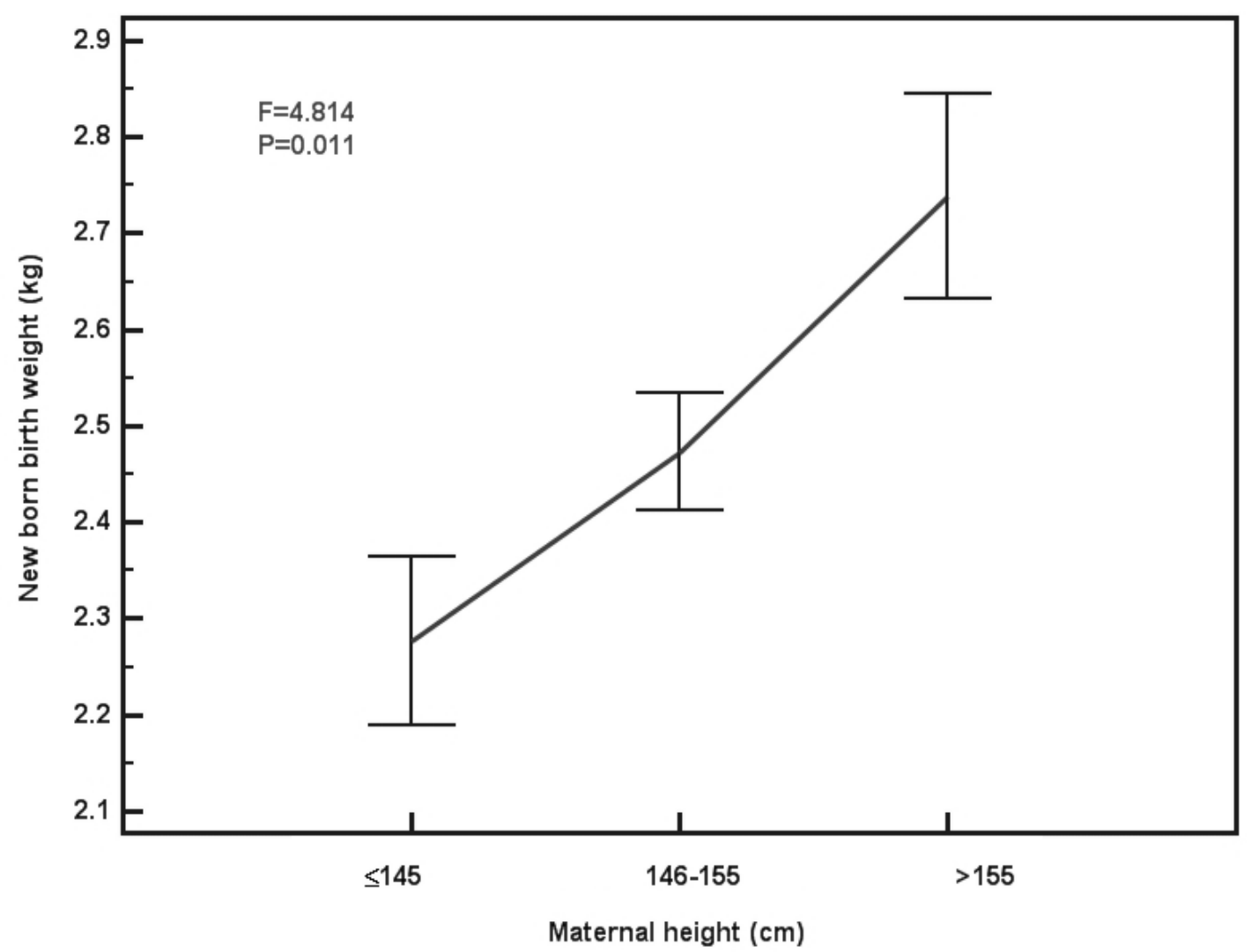

Figure 1. Mean (Se) Birth Weight by Maternal Height, South Kolkata, 2004.

Table 1. Maternal and ne wborn anthropome tric characteristics, South Kolkata, 2004.

\begin{tabular}{|c|c|c|c|c|}
\hline Characteristics & Mean & $\mathrm{SD}$ & Minimum & Maximum \\
\hline \multicolumn{5}{|l|}{ Maternal: } \\
\hline Weight (kg) & 46.0 & 5.9 & 32.3 & 59.3 \\
\hline Height $(\mathrm{cm})$ & 149.4 & 6.0 & 128.9 & 166.4 \\
\hline Head circu mference $(\mathrm{cm})$ & 51.9 & 1.5 & 48.4 & 56.8 \\
\hline MUAC (cm) & 22.5 & 1.9 & 18.2 & 27.1 \\
\hline Biceps (mm) & 3.7 & 1.3 & 2.0 & 7.0 \\
\hline Triceps (mm) & 8.6 & 3.2 & 3.0 & 16.0 \\
\hline $\operatorname{BMI}\left(\mathrm{kg} / \mathrm{m}^{2}\right)$ & 20.6 & 2.2 & 15.6 & 27.9 \\
\hline \multicolumn{5}{|l|}{ Newborn: } \\
\hline Birth weight (g) & 2474 & 409 & 1281 & 3441 \\
\hline Birth length $(\mathrm{cm})$ & 47.3 & 2.1 & 40.7 & 51.3 \\
\hline
\end{tabular}

Linear regression analyses of neonate weight and length as dependent variables revealed that in both cases, maternal height had the most significant impact. It explained $12.9 \%$ (birth weight) and $16.1 \%$ (b irth length) of variation. Maternal weight and head circumference also had significant impact on neonate weight and length. It appeared that none of the other maternal anthropometric characteristics had significant effect on the two dependent variables (Table 2) 


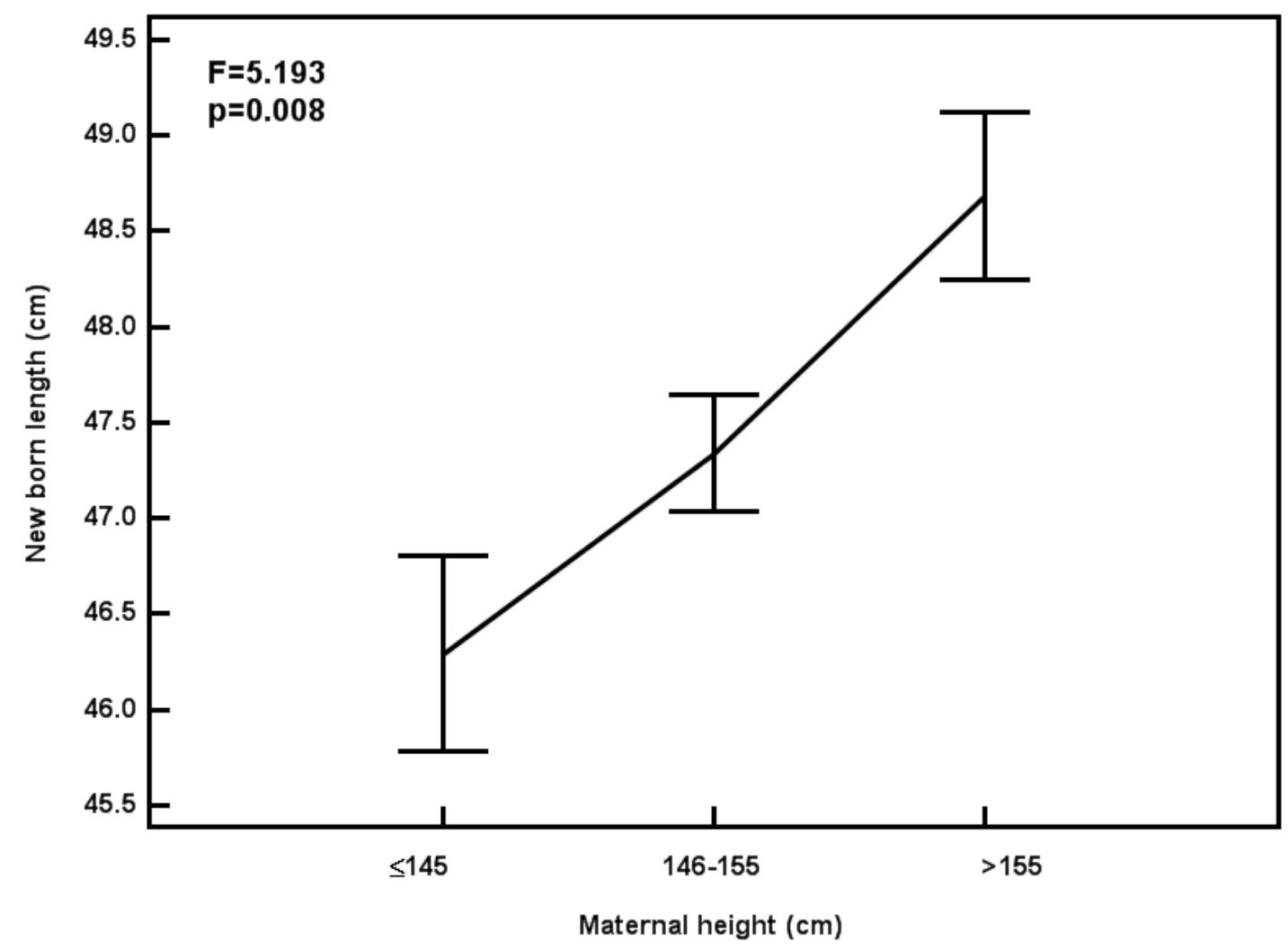

Figure 2. Mean (se) Birth Length by Maternal Height, South Kolkata, 2004.

Table 2. Regression analyses of maternal anthropometry on ne wborn size, South Kolkata, 2004.

\begin{tabular}{|c|c|c|c|c|c|c|c|}
\hline $\begin{array}{l}\text { Dependent } \\
\text { Variables }\end{array}$ & $\begin{array}{l}\text { Independent } \\
\text { Variables }\end{array}$ & B & $\mathrm{SeB}$ & Beta & $\mathrm{F}$ & $\mathrm{P}$ & $\operatorname{Adj}^{2}$ \\
\hline \multicolumn{8}{|c|}{ Birth weight } \\
\hline & ight & 0.02299 & 0.008 & 0.335 & 8.886 & 0.004 & $10.0 \%$ \\
\hline & ght & 0.05590 & 0.008 & 0.375 & 11.480 & 0.001 & $12.9 \%$ \\
\hline & d cir. & 0.07345 & 0.032 & 0.261 & 5.118 & 0.027 & $5.5 \%$ \\
\hline & JAC & 0.02763 & 0.025 & 0.133 & 1.255 & 0.266 & $0.4 \%$ \\
\hline & eps & 0.06345 & 0.037 & 0.202 & 2.969 & 0.089 & $2.7 \%$ \\
\hline & ceps & 0.00574 & 0.015 & 0.044 & 0.138 & 0.711 & $-1.2 \%$ \\
\hline & & 0.02290 & 0.022 & 0.126 & 1.127 & 0.292 & $0.2 \%$ \\
\hline \multicolumn{8}{|c|}{ Birth length } \\
\hline & & 0.09724 & 0.039 & 0.283 & 6.077 & 0.016 & $6.7 \%$ \\
\hline & & 0.14200 & 0.037 & 0.415 & 14.576 & 0.000 & $16.1 \%$ \\
\hline & cir. & 0.35200 & 0.163 & 0.249 & 4.626 & 0.035 & $4.9 \%$ \\
\hline & & 0.05756 & 0.125 & 0.055 & 0.213 & 0.646 & $-1.1 \%$ \\
\hline & & 0.23700 & 0.187 & 0.150 & 1.621 & 0.207 & $0.9 \%$ \\
\hline & & 0.01932 & 0.077 & 0.030 & 0.062 & 0.804 & $-1.3 \%$ \\
\hline BN & & 0.03352 & 0.109 & 0.037 & 0.094 & 0.760 & $-1.3 \%$ \\
\hline
\end{tabular}




\section{DISCUSS ION}

The mean maternal height and weight in this study were similar to those reported by the WHO (7) collaborative study conducted in Pune and Hyderabad, India. The mean maternal head circumference in the present study was similar to that reported in a recent study from Pune, India (17).

Most importantly, this study provided strong evidence that maternal height had the strongest significant impact on newborn size (birth weight and birth length). It is well established that socio-economic status and ethnicity influences height. Stunting is a consequence of long-term poor nutritional intake and is the best indicator of decreased growth in children over an extended period. Stunting has been associated with poorer cognition and school achievement in later childhood (18). Stunting has also been linked to the perpetuation of the cycle of undernutrition by causing low birth weight among offspring of the stunted mother (19). The height of the mother is a wellknown predictive index of perinatal mortality and morbidity. A study from India reported high incidence of LBW (29.\% vs $24.2 \%$ ) infants in mothers with height less than $145 \mathrm{~cm}$ than the mothers with greater than $145 \mathrm{~cm}$ (5). They had 1.32 times risk of giving birth to LBW in comparison to mothers $>145 \mathrm{~cm}$ in height. Several other studies have reported that short stature mother had greater risk for adverse pregnancy outcome $(11,14,20)$.

Thus, this study clearly indicated the strong influence of maternal height on neonate size among adolescent Bengalee mothers. Maternal height alone accounted for $12.9 \%$ and $16.1 \%$ variation in neonate weight and length, respectively. This strong association could have serious health implications among adolescent mothers in the developing country where most of them suffer form prolonged nutritional deficiency. However, since this is a preliminary finding, it needs validation using a larger sample of adolescent Bengalee mothers. If validated, an appropriate maternal height cut-off point for neonate size could be derived. Based on this cut-off point, high-risk adolescent pregnancies can be identified for appropriate nutritional intervention.

\section{ACKNOWLEDGEMENTS}

The author would like to thank to all the subjects who participated in this study. The hospital and the Society for Applied studies staffs are also gratefully acknowledged. Rajiv Gandhi National Fellowship, UGC, India, Society for Applied Studies Trust,
Kolkata, India and Institute of Development Studies Kolkata (IDSK), Kolkata, India spons ored the study.

\section{REFERENCES}

1. World Health Organization. The incidence of low birth weight: an update. Wkly Epidemiol Rec, 1984; 59: 205-211.

2. Edouard L. The epidemiology of perinatal mortality. World Health Stat. Quart, 1981; 39: $289-301$.

3. UNICEF-Indian Council of Medical Research: Birth weight: A major determinant of child survival. Indian J Pediatr, 1987; 54: 801 - 805.

4. Kramer MS. Determinants of low birth weight, Methodological assessment and meta-analysis. Bull World Health Organ, 1987; 65: 663-737.

5. Kamaladoss T, Abel R, Sampath Kuman V. Epidemiological co-relates of low birth weight in rural Tamil Nadu. Indian J Pediatr, 1992; 59: 299-304.

6. Pachauri S, Marwah SM and Rao NSN. A multifactorial approach to the study of the factors, influencing birth weights in the urban community of New Delhi. Indian J Med Res, 1971; 59: 13181341.

7. World Health Organization. Maternal anthropometry and pregnancy outcome: a WHO collaborative study. Bull World Health Organ, 1995; 73 (Suppl): 1-98.

8. Krishna M, Bhatia BD, Gupta J, et al. Predicting low birth weight delivery using maternal nutritional and uterine parameters. Indian $J$ Matern Child Health, 1991;2(3):87-91.

9. Baird D. Social class and foetal mortality. Lancet, 1947; ii: 531-535.

10. Barros FC, Victoria CG, Vaughar JP and Estaislau HJ. Perinatal mortality in Southern Brazil: A population based study of 7392 births. Bull. World Health Organ, 1987; 65: 95-204.

11. Gopalan S. Identification and managemnt of highrisk pregnancies. In: Obstetrics and Gynaecology for Postgraduates (eds SS Ratnam, K Bhaskar Rao \& S A rulku maran. Orient Long man: Madras, 1992; 1: 9-19.

12. Ghosh S, Hooja V, Mittal SK and Verma RK. Biosocial determinants of birth weight. Indian Pediatr, 1977; 14: 107-114.

13. Deshmukh JS, Motghare DD, Zodpay SP and Wadhva SK. Low birth weight and associate maternal factors in an urban area. Indian Pediatr, 1998; 35: 33-36.

14. Bhatia BD, Tyagi NK, Handa P. Relationship of low birth weight with anthropometry and maternal 
height-weight indices. Indian J Med Res, 1985; 82: 374-376.

15. Verma V and Das KB. Teenage Primigravidae. A comparative study. Indian J Public Health, 1997; 41 (2): 52-55.

16. Lohman TG, Roche AF and Martorell R. Anthropometric standardization references manual. Champaign, Illinois: Human Kinetics Books. 1988

17. Leary S, Fall C, Osmond C, Lovel H, Campbell $\mathrm{D}$, Eriksson J, et al. Geographical variation in relationships between parental body size and offs pring phenotype at birth. Acta Obstet Gynecol, 2006; 85 (9):1080-1089.
18. Chang SM, Walker SP, Grantham-McGregor S, et al. Early childhood stunting and later behavior and school achievement. $J$ Child Psychol Psychiatry, 2002; 43: 775- 783.

19. Kramer MS, Olivier M, McLean FH, et al. Determinants of fetal growth and body proportionality. Pediatrics, 1990; 86: 18-26.

20. Pickett KE, Abrams B, Selvin S. Maternal height pregnancy weight gain and birth weight. Am J Hum Biol, 2000; 12: 682-687. 